\title{
INTEGRATED DYNAMIC MANAGEMENT OF VIRTUAL ENTERPRISES. CONCEPTS AND SOLUTION IN THE BIDSAVER PROJECT
}

\author{
Roberto Santoro \\ CE Consulting,e-mail: roberto.santoro@ceconsulting.it \\ Bruno Lisanti \\ CE Consulting, e-mail: bruno.lisanti@ceconsulting.it \\ Luca Spotorno \\ CE Consulting, e-mail: luca.spotorno@ceconsulting.it
}

ITALY

\begin{abstract}
This paper is targeted upon the new methods and infrastructures for the dynamic management of Virtual Enterprises (VE), as built up in the BIDSAVER Project (IST Project \#10768) part of the VIVE (VIrtual Vertical Enterprise) initiative, headed by $C E$ Consulting.

The VIVE initiative formulates a methodological and ICT framework, enabling Small and Medium Enterprises (SMEs) to join their forces to race on the global market as if they were a single, larger Company. Such a framework is based on the concept of the Business Integrator (BI), an external entity providing Companies with both management and methodological support, and in charge of coordinating the Virtual Enterprise activities throughout its lifetime.

The ICT infrastructure is made up of modules developed by customizing existing software suites, which exploit XML-based interfaces.
\end{abstract}

\section{INTRODUCTION}

The objective of this paper is to describe methodologies and tools for the dynamic management of Concurrent/Virtual Enterprises, as developed in the BIDSAVER Project (IST Project \# 10768), led by CE Consulting. The BIDSAVER Project aims at providing a new professional entity, the "Business Integrator" as identified in the VIVE Project, with an ICT infrastructure, suitable to describe dynamically Product, Process and Organization of the Virtual Enterprise, to support the search through the web for potential VE Partners which best suit with VE contingent needs, and to support the co-ordination and control of the progress on the work achieved by the Partners.

This is achieved by delivering to the Business Integrator an Information System, composed by three integrated management modules, devoted respectively to the management of VE data, to the web-based search of partners, and to the integration of operational functions.

\footnotetext{
The original version of this chapter was revised: The copyright line was incorrect. This has been corrected. The Erratum to this chapter is available at DOI: 10.1007/978-0-387-35585-6_68
} 
The ICT infrastructure allows therefore for providing partner Companies with management and methodological support to share risks and benefit of the business venture. The system is developed by adapting existing software solutions to the new proposed business model, focused on the cooperative process and on the coordination needs.

In this scenario, the availability of an information capturing agent, based on XML technology and on an advanced searching mechanism (tailored on industrial profile pattern search more than generic keywords) is one of the most significant innovations of the BIDSAVER project.

\section{THE VIVE VIRTUAL ENTERPRISE SCENARIO}

All over Europe, seventeen millions of Small and Medium Enterprises (SMEs) give a substantial contribute to the global economic system.

The extraordinary development of the Information and Communication Technology (ICT) allows for innovative relationship among enterprises, geographically dispersed and organised in extended networks and for overcoming their traditional business scheme, based on statically established co-operations and enabled by personal relationships and proximity of companies locations.

As a matter of fact, that approach, even though effective in the past years scenario, is showing its limits in the capability of facing the requirements of the global market, where the search for competitiveness cannot be limited by geographical and personal constraints.

During IV European RTD framework program, the VIVE Project, coordinated by CE Consulting, has contributed significantly to the formalization of advanced, co-operative schemes among geographically dispersed Enterprises. This was achieved by introducing a new entity, the "Business Integrator", suitable to support the constitution and operation of Virtual Enterprises grouping SMEs around a specific business opportunity.

The Business Integrator would:

- support the structuring of identified business opportunities,

- specify the required business processes, the enterprise integration infrastructure (communications, information sharing means, ...), the skills and the capacities needed by the Virtual Enterprise to be created,

- solicit and support suitable SMEs to enter the Virtual Enterprise.

\section{THE BUSINESS INTEGRATOR IN THE BIDSAVER SCENARIO}

Cooperation among SMEs in a Virtual Enterprise is to be performed only according to business opportunities and to purely competitiveness-oriented criteria. This implies the constitution of Virtual Enterprises with a strong dynamic connotation, characterized by the possibility of selecting an optimized Partners' set.

In the constitution and operation of a Virtual Enterprise, the Business Integrator must therefore adopt a business-centric approach. In this respect, the Virtual 
Enterprise is the equivalent of a set of projects, managed through the continuous search of best capacities and capabilities, where selection and management of resources is performed both at the level of individual SMEs and at the aggregation level of Virtual Enterprise.

However, the Business integrator is confronted by several major issues:

- The limited functionality of current SW systems to satisfy the Business integrator management functions. In particular the ability to support the Virtual Enterprise decision process and conflict resolution by the possibility of on line consultation of the project status by any of the participating actors. Each partner should also be able to directly verify possible impacts on themselves, other affected partners and the overall project deriving from their performance and suggested actions. These impacts would be described in terms of alternative cost functions.

- The lack of reference code of practice for the co-operation process definition and especially the absence of standardized "Business Extended Mark-up Language", capable of defining computer processable information on current capacity and capability of potential partners, resulting in limited efficiency in constituting the VE. The vision would be a "Plug and Play" search in the web for co-operation- ready partners addressing specific requirements of an individual business.

In addition to this, the Business Integrator should also consider the lack of a legal framework for the Virtual Enterprise, capable to regulate rights and responsibility among the partners, which operates through the web. Issues such as Intellectual Property Rights, Product Liabilities, Defaulting partners' responsibility and in general contractual terms and conditions, assume a new dimension of complexity due to the dynamic and ephemeral nature of the Virtual Enterprise and its innovative, operating way.

\section{CURRENT RESULTS}

The objective of BIDSAVER is to contribute significantly to the resolutions of the previously mentioned issues, through the development and assessment of a framework for the constitution and operation of a Virtual Enterprise with the participation of SMEs searched through web-based information agents, organized according to best fit criteria and regulated by a validated legal framework.

The Information System developed to support the Business Integrator's function is composed by three integrated management modules:

- a Business Breakdown Structure (BBS) management module, allowing the management of evolution of VE through a model (BBS) that accommodates cross mapping of product physical/functional items, tasks and associated resources/risks/timing, and responsible entities/ organizations with associated contractual elements

- An information capturing agent, responsible for the actual search on the web of potential partners (to be dynamically organised in the Virtual Enterprise according to best fit criteria) and for providing updated information on co-operation opportunities 
- The Business information integration module, integrating operational functions, that leverage on e-Commerce and Internet technology, and on pre-selected commercial solutions to be configured according to VE requirements

The ICT strategy pursued by BIDSAVER exploits the availability of ICT solutions that cover specific areas of requirements, and which are customized and integrated according to VE models.

The Information and Management System obtained is tested on two actual business cases, the first relevant to a Virtual Enterprise for the design of Microsatellites, the second relevant to a Virtual Enterprise for the optimisation of a supply chain in the mechanical components sector. The two business cases have been selected as they present complementary characteristics from a management point of view. This is expected to give a quite exhaustive validation of the ICT infrastructure design, as it will allow for testing a wide variety of operational and functional requirements.

In line with the conceptual structure of VE models, the ICT environment is designed by exploiting best available technology and solutions, pursuing the use of XML-based interfaces in order to support the re-deployment over different sets of commercial solutions.

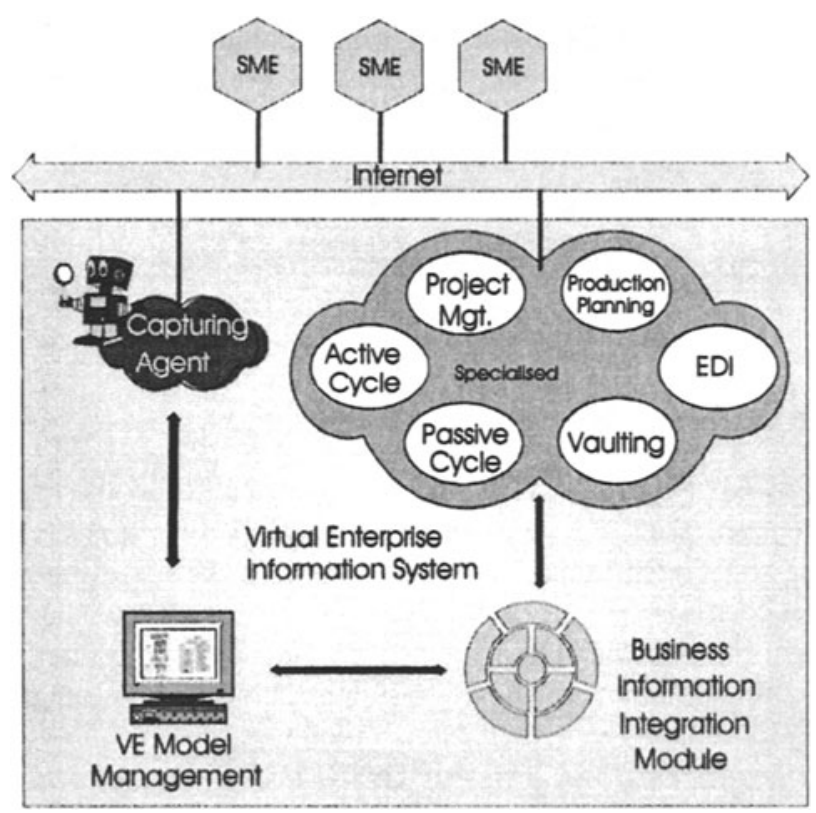

Figure 1 - VE Information System concept

The ICT infrastructure was designed starting from the formalization of the Virtual Enterprise's models. Such models contain the information required to describe the Virtual Enterprise's status in terms of product information and configuration, tasks and overall process status and VE's actual organization. In addition, a full description of the operating procedures and management processes 
(Partner search process, data management and control, information flow formalisation and so on), suitable to facilitate the evolution of the Virtual Enterprise's status, was given for the two identified business cases.

The ICT architecture was designed to:

- integrate legacy, purchased and internally developed applications, regardless of their technologies

- include a number of value-added services (flow control, transformation, message definition, message warehousing)

- support both the publish/ subscribe and request/reply styles of application interaction, as well as reliable and/or guaranteed message delivery

- be centrally managed and administered

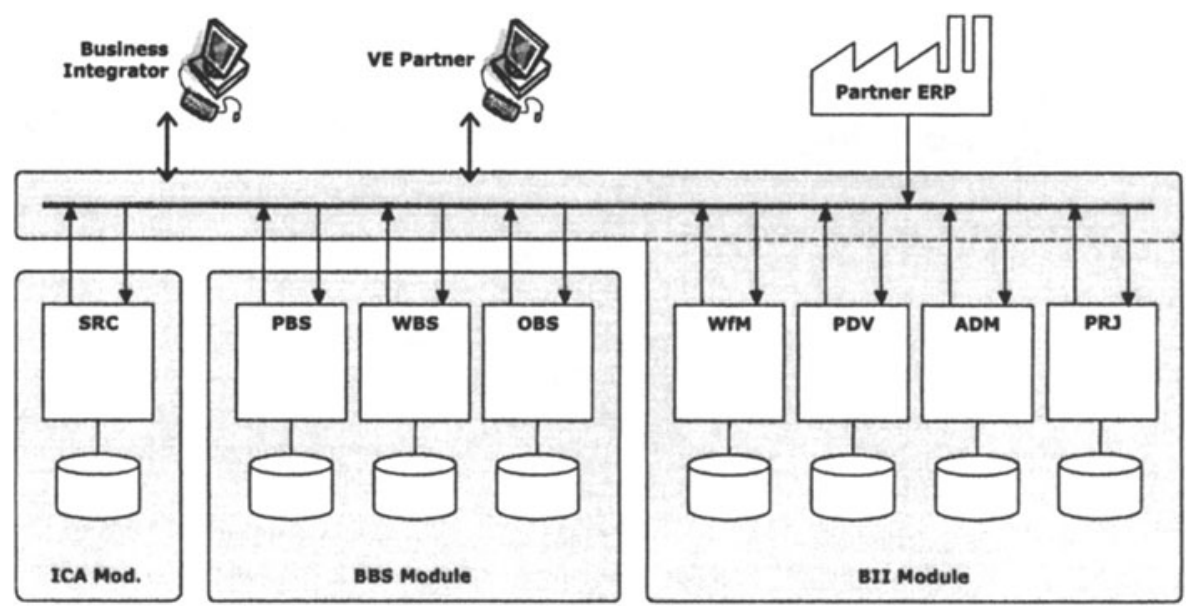

Figure 2 - Conceptual structure of modules

The BBS (Business Breakdown Structure) management SW module takes charge of managing the vision, common to all VE partners, of the overall project, of the assigned responsibilities and of the impact that each activity has on the other activities.

The information to be controlled spans over several views: structure and description of the product; organisational structure and the related responsibility assignments; operational plan; network of interconnections between views.

The module is based on a centralised repository, which stores all the operational information accessible, by the SMEs at various level of privilege. At any time each enterprise can retrieve information on the project (according to its privilege level) in order to know the impact of possible changes through the availability of cost and time functions, possibly provided through commercial modules.

The second major management module is the one devoted to Company information capturing. The main task of the agent is to find in the partner expertise domain the best candidate to become a member of the Virtual Enterprise.

The agent module architecture is based on: 
- Agent Monitoring Server (AMS). The AMS holds a list of all the active Information Capturing Agents, contains the most recently updated partners' domain model and keeps tracks of global information (such as the number of SMEs in the VE) for the purpose of activity co-ordination. The AMS can share information with each information-capturing agent or can allow two or more agents to do this, adopting the XML data format exchange. The AMS is also in charge of extracting and managing the subset information from the BBS instance that specify the requirements to partners and guide the investigation and search among candidate SMEs.

- Information Capturing Agents, one for each enterprise. The main task of the agent is to automatically collect the necessary data from the enterprise internal resources (web site, informative systems, local database). All the data are stored in a profile used to present a suitable interface of the enterprise to other enterprises in the partner expertise domain. The agent also can collaborate with agents from other enterprises, in order to obtain the necessary information for searching potential partners to constitute a VE. Data exchange among the agents is in the XML format, allowing a definition of flexible data types with a www interface.

The choice of Java programming language for the Capturing Agent allows any computer with a Java Virtual Machine installed (regardless of the operating system) to run the program.

The last major management module is the Business Information Integration module. This module constitutes the glue of the VE Information system. It is in charge of generating from the BBS module the technological views that constitute the interfaces to selected functional applications (Project Management and so on), and to manage the interoperability among applications through the establishment of rules that accommodate VE operational concept. The Business Information Integration Module accommodate the interfacing with specialized modules, implementing the fundamental functions identified in the operational concept of the VE, and including Project Management, Production scheduling, Information vaulting and reporting, Management of VE active and passive cycle.

The module is the basis for the creation of the operational section of the Virtual Enterprise Information System:

- built to leverage on e-Commerce and Internet technology to accommodate low cost access to information and functions by partners,

- based on commercial solutions and configured according to VE requirements;

- improved through add-ons accommodating specific functions necessary to the successful VE operation.

\section{TECHNOLOGICAL SOLUTION}

The major commercial elements that constitute the BIDSAVER prototype ICT environment are:

- the UGS iMAN system, allowing for the product data management support and constituting the reference repository manager for the project 
- the Microsoft Project 2000 (and associated web server application Project Central) accommodating main Project Management functions

- the Heletel XEOS search system, accommodating the agent based search for information on the web.

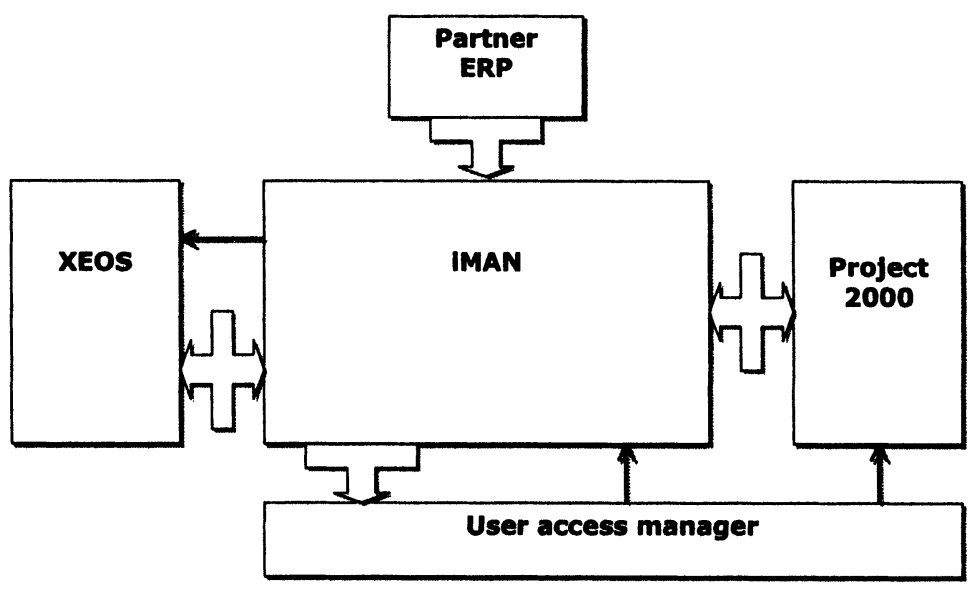

Figure 3 - Main Commercial Solutions

The openness of the system to accommodate alternative commercial solutions that are already available to possible service suppliers is pursued through the clear identification of boundaries at conceptual modules level and at commercial solution level.

The procedure used is constituted of three main steps:

- mapping functions associated to conceptual modules on the commercial systems (used also in the identification of suitable commercial solutions)

- specifying and enacting the interfacing between commercial modules through an XML based approach

- specifying the actual interfaces between conceptual modules when occurring within a single commercial module.

All the commercial solutions adopted support the web-based operation, either through standard mechanisms or through Virtual Private Networking technology for most security critical functions.

The scalability of technological solutions is one of the main issues for the system, and the implementation costs call up for an approach that exploit the concept of "pay-per-use" for SMEs, through the constitution of local Service Centres that would act as trusted interface to SMEs, and perform as nodes of a wider network of service centres to allow for effective participation of SMEs to the global market opportunities.

The constitution of those Service Centres is today's challenge for the VIVE initiative, and is capable of offering an innovative mechanism for cooperative working both for the exploitation of Virtual Enterprising concepts and for the successful implementation of Extended Enterprising approach in the supply chains of large enterprises and international consortia. 


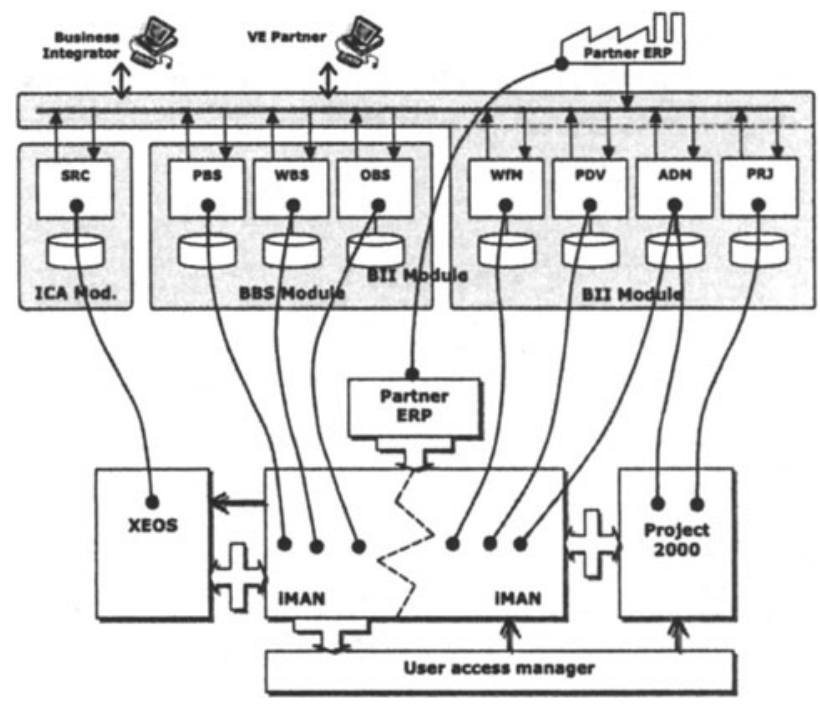

Figure 4 - Mapping functions and interfaces

\section{BIDSAVER CONSORTIUM AND INTEREST GROUP}

BIDSAVER brings together all the required skills to achieve project objectives and maximise the potential for exploitation of project results: a Methodology Partner, a legal firm, three ICT partners, three industrial companies for the $1^{\text {si }}$ Pilot Case (devoted to the development of micro-satellites), and three industrial companies for the $2^{\text {nd }}$ Pilot Case, addressing a mechanical equipment supply chain.

The dissemination of the BIDSAVER experience and results is one of the most crucial tasks of the Project. The strategy pursued by the partners is mainly focused on the further development of the Virtual Community of VIVE potential users (http://www.vive-ig.net), which was created within the VIVE Project frame.

As a matter of fact, the number of VIVE Community members is rapidly increasing (registered members are about 370 from 275 companies/organizations as of October 2001).

The VIVE Community members belong to 18 different European Countries (even outside the European Union); they are mainly SMEs, but also SMEs associations and Regional Centres are significantly represented.

\section{REFERENCES}

1. Santoro Roberto, Conte Marco, The dynamic Constitution and Management of Virtual Enterprises, ICE 2000 proceedings.

2. Conte, Marco: VIVE ESPRIT Project 26854 Public Final Report, 2000

3. Stokes Chris: Competing by numbers - Innovation and Technology Transfer, Vol 4/00, July 2000 